\title{
Aprendizaje basado en proyectos: una propuesta eficaz para el desarrollo de las competencias en el master de Acuicultura
}

\author{
Silvia Martínez-Llorens ${ }^{\mathrm{a}}$, Ignacio Jauralde García, David S. Peñaranda, Ana Tomás-Vidal, Miguel \\ Jover Cerdá \\ ${ }^{a}$ Departamento de Ciencia Animal, Universitat Politècnica de València, Camino de Vera, 14, València, 46071, \\ Spain; silmarl1@dca.upv.es, igjaugar@upv.es, dasncpea@upv.es, atomasv@dca.upv.es, mjover@dca.upv.es.
}

\begin{abstract}
This work shows an of Project Based Learning experience on Interuniversity Master of Aquaculture degree at the Universitat Politécnica de València. The ABPr focused on the development of a fish farm project, in which the students could acquire a combined vision of the subjects taught during the master's degree, promoting the connection between subjects, thus reinforcing the interdisciplinary character of the project. To evaluate the methodology, a quantitative and a qualitative survey was conducted with the aim to know the students opinions. The students have positively valued the methodology, highlighting the importance of the project developed for their professional training, as well as the fact that the project carried out as teamwork met their expectations regarding the teachers'work. Although no negative evaluations about the developed methodology were observed, some aspects such as time scheduling, and the integration of additional subjects/ disciplines should be improved.
\end{abstract}

Keywords:Project-based learning, Master of Aquaculture, teamwork, student motivation

\section{Resumen}

En el presente trabajo se muestra una experiencia de la aplicación del Aprendizaje Basada en proyectos en la titulación del Master interuniversitario de Acuicultura de la Universitat Politécnica de València. El ABPr se centró en el desarrollo de un proyecto de una piscifactoría, en el cual los alumnos pudieran adquirir una visión en conjunto de las asignaturas impartidas en el máster, fomentando la conexión entre ellas, reforzando, por tanto, el carácter interdisciplinar del proyecto. Para evaluar la metodología se realizó cuestionario cuantitativo y otro cualitativo con el objetivo de conocer las opiniones de los alumnos. Los alumnos han valorado positivamente la metodología, destacando la importancia del proyecto desarrollado para su formación profesional, así como también se valoró sustancialmente que el trabajo desarrollado en grupo cumplió sus expectativas en cuanto a la labor de los docentes. Aunque no se observaron valoraciones negativas de la metodología desarrollada, se deben de mejorar algunos aspectos como la planificación del tiempo y consolidar relaciones de integración o la interacción entre diferentes disciplinas/asignaturas.

Palabras clave: Aprendizaje basado en proyectos, master de acuicultura, trabajo en grupo, motivación del alumnado

\section{Introducción}

El máster interuniversitario de acuicultura, el cual se imparte desde el curso 2006/2007, es un master de un año de duración, de cuya docencia se encargan la Universitat Politècnica de València junto con la 
Universidad de Valencia y con el Instituto de Acuicultura de Torre de la Sal (CSIC). El número de alumnos por curso en este master se encuentra entre 20 y 25 y su perfil de ingreso es muy variado, ya que pueden proceder de varias titulaciones, unas con diferentes bases técnico-científicas, como por ejemplo licenciado en biología, ingeniería agronómica, veterinaria, farmacia, ciencias del mar, ingeniería de montes, y todos aquellos nuevos grados que sustituyen a las anteriores licenciaturas o bien otras titulaciones procedentes de Sudamérica, como ingeniería zootecnista o ingeniería pesquera.

En referencia a las asignaturas que se imparten en la UPV, éstas tienen un marcado enfoque práctico, como "Diseño y gestión de piscifactorías", "Ingeniería de sistemas", "Nutrición y alimentación" y "Producción de especies continentales y tropicales". Dada la naturaleza de estas asignaturas, los profesores siempre han querido acercar a los estudiantes a situaciones lo más cercanas posibles al contexto profesional en que éstos desarrollarán en el futuro, por lo que se pretende que la enseñanza tenga lugar en el contexto del mundo real o de la práctica profesional, como se recomienda en la aplicación de metodologías activas.

\subsection{Visión individualizada de las asignaturas}

Concretamente, en el master de acuicultura, las asignaturas impartidas tienen una patente relación entre ellas. Sin embargo, los profesores del master han observado cómo los alumnos tienen una visión individualizada de las asignaturas, los cuales, en la gran mayoría de casos, se limitan a aprobarlas y estudiarlas sin buscar o experimentar la interacción existente entre las mismas. Este hecho, limita la posibilidad de obtener una visión más real y general de situaciones y problemáticas reales de la acuicultura. En este sentido, la asignatura de Diseño y Gestión de piscifactorías permite englobar todas estas disciplinas y asignaturas, durante el desarrollo del proyecto de una instalación de acuicultura que tienen que llevar a cabo en la asignatura, se consideran aspectos tanto desde el punto de vista de diseño como nutricional, de biología y zootecnia de las diferentes especies acuícolas, económico, de ingeniería de las instalaciones, manejo de especies y de piscifactorías, impacto ambiental, bienestar de los animales,... de modo que se pueden aplicar todas estas disciplinas mediante el desarrollo de este proyecto, pudiendo adquirir una visión en conjunto de las asignaturas impartidas y la conexión entre ellas.

\subsection{Solución: ABPr}

El ABPr es una herramienta metodológica ampliamente utilizada, tanto en la educación la educación superior y universitaria (Sánchez, 2013). El ABPr implica formar equipos conformados por personas con perfiles diferentes, áreas disciplinares, profesiones, idiomas y culturas que trabajan juntos para realizar proyectos con el propósito de solucionar problemas reales (Reverte Bernabeu, Gallego Sánchez, MolinaCarmona, \& Satorre Cuerda, 2007) siendo el proyecto la finalidad principal (Sánchez, 2013), por ello, tiene una gran aplicación en los estudios de ingeniería, tal y como se muestra en previos estudios (Bermell, Marton, Villanueva, Sánchez, \& Carlos, 2019; Estruch \& Silva, 2006; Fernández \& Duarte, 2013; López \& Vázquez, 2015; Vega, Portillo, Cano, \& Navarrete, 2014)(Bermell et al., 2019; Rodríguez-Sandoval, Cortés-Rodriguez, Vargas-Solano, \& Luna-Cortés, 2010; Vega et al., 2014). Una docencia basada en proyectos por grupos tiene unos altos requerimientos de comunicación y de intercambio de información (Reverte Bernabeu et al., 2007). En concreto, los alumnos del master de acuicultura proceden tanto de diferentes nacionalidades (España, Colombia, Francia, Italia, Perú, Chile, Venezuela, Egipto, Angola), como de diferentes titulaciones, por lo tanto, de áreas disciplinares diferentes y deben trabajar conjuntamente. Mediante la aplicación del ABPr en el contexto del máster de acuicultura, los alumnos deben ser capaces de desarrollar un proyecto de una piscifactoría, en el cual se 
incorporan los conceptos impartidos en diversas asignaturas del Master de Acuicultura. En concreto, el presente estudio se engloba dentro de un proyecto docente, que pretende potenciar las competencias de aplicación y pensamiento práctico, análisis y resolución de problemas, innovación, creatividad y emprendimiento, diseño y proyecto, comunicación efectiva, trabajo en equipo, así como planificación y gestión del tiempo.

Por todo ello, el aprendizaje basado en proyectos colaborativos puede ser de gran utilidad para mejorar la visión conjunta de las distintas disciplinas que se pueden aplicar en acuicultura, con el fin de que los estudiantes puedan hacer frente a problemas reales, generalmente interdisciplinares, que deben estructurar, organizar y esforzarse, con ayuda del profesorado, por encontrar soluciones con sentido. De este modo se fomentará la formación de profesionales que trabajan en equipo en lugar de aplicar métodos que solo los preparan para trabajar de forma individualizada y con una visión restringida de los problemas.

\section{Objetivo}

En el aprendizaje basado en proyectos, la labor del profesor no se limita a la exposición de las asignaturas, sino a conducir, orientar y guiar a los alumnos en el proceso de toma de decisiones, de manera que cada equipo o grupo pueda desarrollar su tarea de forma autónoma (Rodríguez-Sandoval et al., 2010). Es por ello, que el papel del profesor a lo largo de todo el proceso va a ser decisivo para avanzar en el aprendizaje y éste va a tener que adaptarse junto con los alumnos a las nuevas metodologías de enseñanza. Debido a que la implantación del ABPr es reciente, es importante conocer la percepción por parte de los alumnos y los profesores del master que han participado en dicho proyecto, de manera que se puedan aplicar mejoras en la metodología aplicada, siendo éste el objetivo del presente estudio.

\section{Desarrollo de la innovación}

Como ya se ha mencionado en el anterior apartado, los alumnos del master de acuicultura abordan diferentes disciplinas con diversas bases biológicas para la producción de organismos acuáticos, adquiriendo los conocimientos correspondientes, pero sin ninguna conexión en común, los cuales tampoco se aplican conjuntamente en la mayoría de los Trabajos finales de master que se realizan, pues éstos suelen ser de materias muy específicas (reproducción, nutrición o patología de especia acuáticas), sin interacción entre ellas.

Por esta razón, se propuso desarrollar el ABPr en una asignatura del segundo cuatrimestre, para asegurar que todas las asignaturas de bases biológicas se hayan impartido durante el primer cuatrimestre, y otras asignaturas como Acuicultura sostenible y Sistemas de producción de especies continentales, simultaneas a la asignatura de diseño, colaboran durante el desarrollo del proyecto. El proyecto en cuestión se centró en el diseño de una piscifactoría: desde la planificación zootécnica (Jover, Martínez, Tomás, \& Pérez, 2016; Merinero, Llorens, Vidal, \& Cerdá, 2016), el dimensionado de la instalación y los equipos, el análisis e implementación de las diferentes alternativas, tanto desde el punto de vista medioambiental (generación de residuos y consumo de agua), como económico. En la Figura 1 se muestra un esquema general de la metodología a desarrollar. En ésta se aprecian las diferentes tareas del proyecto de innovación. En la Tarea 1, se recogen las asignaturas/conocimientos que el alumno debe haber cursado/adquirido de cara a desarrollar el proyecto, dos ellas se desarrollan durante el primer cuatrimestre (Nutrición y alimentación y Bases de Ingeniería de sistemas) y las otras dos simultáneamente al proyecto, durante el segundo cuatrimestre (las cuales colaboran al desarrollo de éste, Acuicultura sostenible y 
Sistemas de producción peces continentales). La variedad de tópicos a tratar y el volumen de trabajo exigido hacen que el alumno desarrolle las habilidades necesarias para el trabajo en grupo, siempre y cuando quieran superar con éxito la asignatura de diseño de instalaciones.

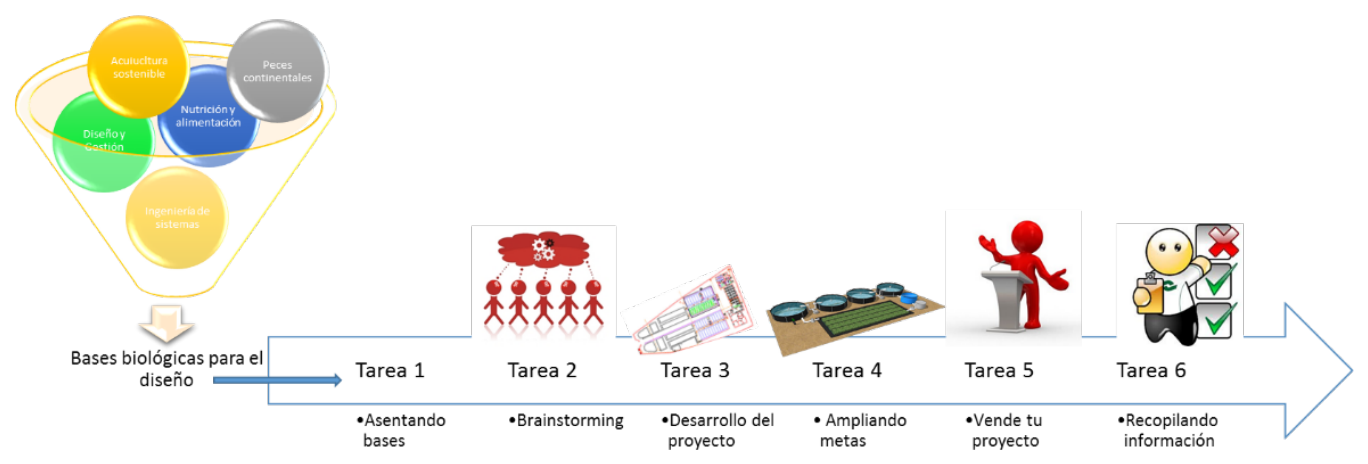

Figura 1: Esquema del desarrollo del PIME

Durante el segundo cuatrimestre se trabajó la asignatura de Diseño y Gestión de instalaciones, que fue la que ensambla todos los conocimientos adquiridos previamente, en la cual se puso en marcha la Tarea 2 "Brainstorming" (Figura 1), que consistió en organizar el aula en grupos de 2 ó 3 alumnos, tal y como recomiendan estudios anteriores (Gayo, Lanvin, Salvador, \& del Río, 2006; Sánchez, 2013), para un adecuado desarrollo del ABPr. Esta tarea se inició una vez impartidas 2 sesiones de tres horas con las bases de diseño de piscifactorías y modelos de crecimiento de peces. Durante esta actividad se pretendía fomentar la participación y la integración de todos los miembros del grupo, democratizarla, y presentarla a todos los presentes en el aula. En esta actividad se definieron las siguientes metas del proyecto:

a. ¿Qué especie vamos a elegir para diseñar la piscifactoría?

b. ¿Qué sistema de producción es el más indicado para esta especie?

c. ¿Hasta dónde podemos llegar en el proyecto con los conocimientos adquiridos previamente? (Nogales Mérida, Jover Cerdá, Martínez Llorens, \& Tomás Vidal, 2011)

d. ¿Qué información hay disponible que nos permita poder desarrollar el proyecto?

El desarrollo del proyecto se efectuó dentro de la Tarea 3 (Figura 1), en la cual se hizo un diseño zootécnico como base para los posteriores cálculos. Este diseño siguió la metodología descrita en trabajos anteriores (Jover et al., 2016), en la que se muestran detalladamente las pautas para el diseño.

De la misma forma, y en esta fase, los alumnos tenían a su disposición videos Polimedia realizados en la asignatura de Diseño y Gestión, donde disponían de un apoyo en el planteamiento del diseño zootécnico. En todo momento esta tarea fue realizada por los grupos de trabajo bajo la supervisión del profesor de forma presencial en prácticas informáticas. Durante toda la fase de elaboración, los profesores implicados realizan el seguimiento de un portafolio por equipo para evaluar la evolución del proyecto.

Una vez realizada y planificada la producción, así como el diseño zootécnico de la piscifactoría, los alumnos aplicaron los conocimientos adquiridos en diversas asignaturas, de manera que ayuden a completar el proyecto de la piscifactoría, esto se llevó a cabo en el marco de la tarea 4 (Figura 1).

En la Figura 2, se muestran qué contenidos de cada asignatura han contribuido para la consecución del proyecto: 


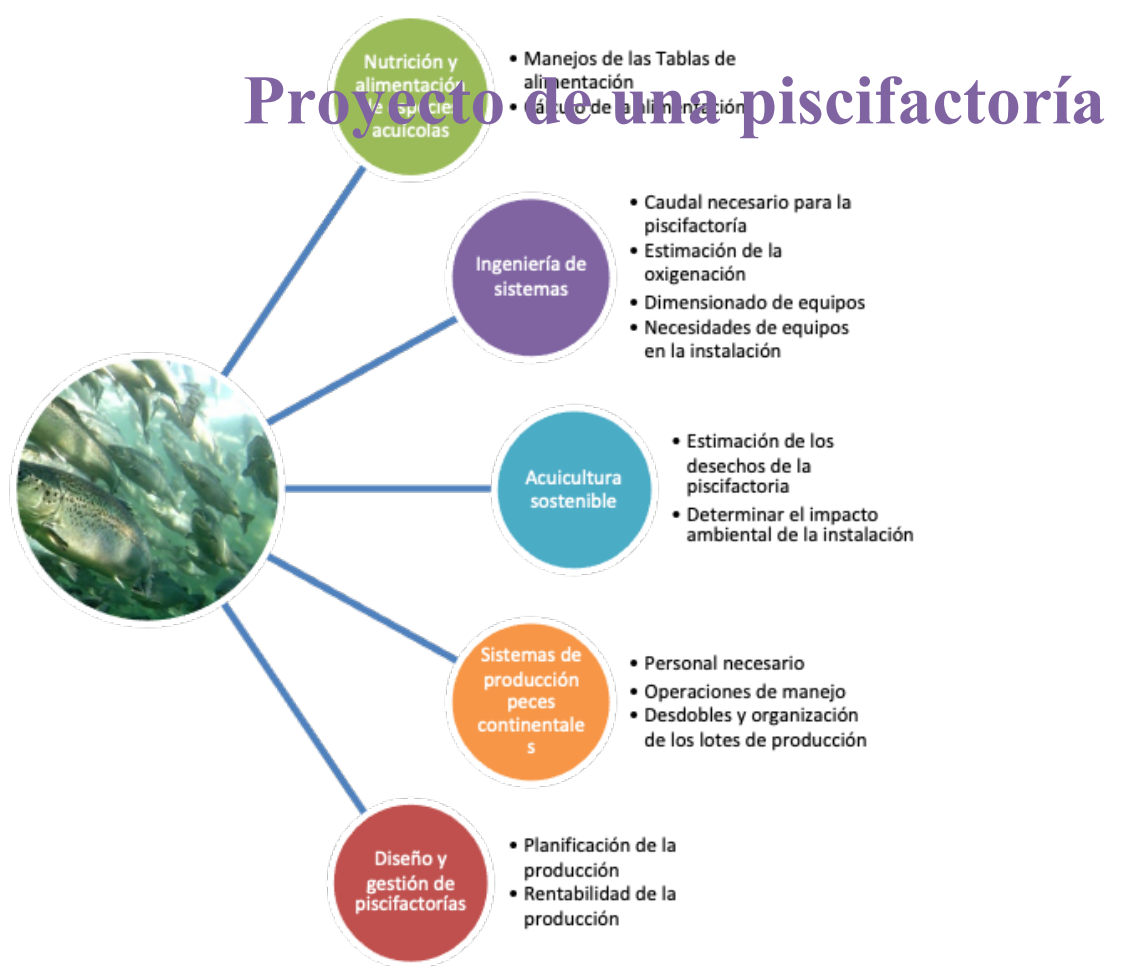

Figura 2: Contribución de los conocimientos adquiridos en cada asignatura del master de acuicultura al desarrollo del proyecto

Una vez finalizado el proyecto de la instalación, los alumnos expusieron su proyecto, dentro de la Tarea 5 "Vende tu proyecto" destacando las ventajas de su proyecto, tanto productivas, como económicas.

Tabla 1. Cuestionario de evaluación de los alumnos sobre el ABPr durante su primer curso de aplicación

\section{Cuestionario sobre el ABPr}

Escala

Q1 ¿Cree usted qué el proyecto plateado es importante para su formación profesional?

Q2 ¿Se aplicaron los conceptos vistos en clase para el desarrollo del proyecto de aula?

Q3 ¿Se aplicaron los conceptos vistos en asignaturas previas para el desarrollo del proyecto de aula?

Q4 ¿Este tipo de metodologías para el aprendizaje satisfacen sus expectativas como estudiante comparándola con otros métodos tradicionales?

Q5iConsidera que esta metodología es útil para aplicar los conocimientos vistos en clase para solucionar problemas del proyecto?

Q6 Usted considera que la orientación para el desarrollo del trabajo por parte del docente cumplió con sus expectativas

Al final del curso, se realizó una evaluación del proyecto del primer año de implantación del proyecto de innovación docente (Tarea 6. Recopilando información, Figura 1). Aquí se consideraron las encuestas docentes por parte de los estudiantes, así como una encuesta específica sobre el ABPr, la cual se muestra en la Tabla 1, para poder implantar mejoras sobre el proyecto en los cursos venideros.

De la misma forma, se realizó una encuesta para determinar qué capacidades consideran adquiridas por parte de los alumnos, la cual se muestra en la Tabla 2. 
Aprendizaje basado en proyectos: una propuesta eficaz para el desarrollo de las competencias en el master de Acuicultura

Tabla 2. Cuestionario de evaluación de los alumnos sobre el ABPr durante su primer curso de aplicación

\section{Cuestionario sobre el ABPr}

Escala

Consideras que las estrategias ABPr son muy adecuadas para desarrollar (entre otras) las siguientes capacidades (cada ítem)

CA. Trabajo en grupo

CB. Aprendizaje autónomo

CC. Participar activamente en el desarrollo del proyecto

CD. Capacidad para Planificación el tiempo

CE. Capacidad de expresarse de forma adecuada

Otros aspectos que le gustaría destacar tanto negativos como positivos

Respuesta abierta

\section{Resultados}

En general, se puede considerar, que los alumnos han valorado muy positivamente la aplicación del ABPr, como se puede observar en la Figura 3, obteniendo una media general superior a 4 sobre 5. Ha habido una excelente participación de los alumnos en el aula durante el desarrollo del proyecto, obteniendo los alumnos, casi en su totalidad una puntuación de notable. También como evidencia hay que destacar que en las encuestas de satisfacción de los alumnos que realiza la UPV, han mejorado las puntuaciones notablemente durante este curso, para la mayoría de las asignaturas, poniendo de manifiesto un incremento en la motivación de las asignaturas. Los trabajos han sido muy bien puntuados y los exámenes han tenido un éxito de aprobados del 100\%.

Las cuestiones mejor valoradas fueron las Q1 y Q6 (Tabla 1), relativas a la importancia del desarrollo del proyecto para su formación profesional y a las expectativas del alumno por la asignatura. Así también, los alumnos han valorado muy positivamente los conocimientos impartidos en clase y desarrollados en el proyecto (respuesta Q2).

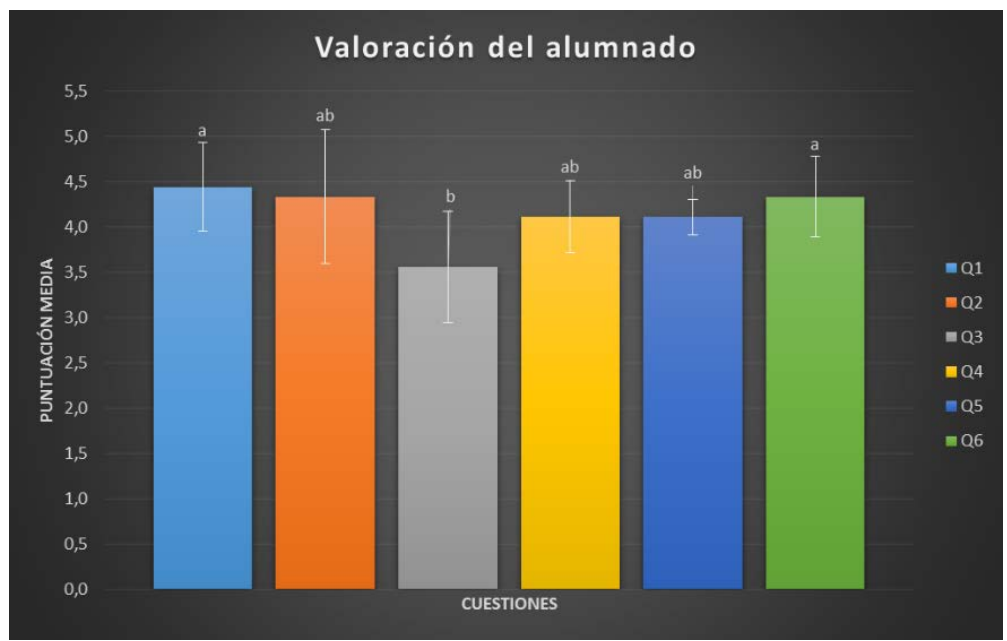

Figura 3. Valoración media de los alumnos, respecto a los objetivos planteados en el ABPr*. Los datos que aparecen en el gráfico son la media \pm desviación estándar $(n=10)$. Letras diferentes en las barras indican diferencias estadísticas significativas con una $P<0,0$.

*Cuestiones planteadas se pueden consultar en la Tabla 1

No obstante, la respuesta que presentó significativamente una menor puntuación, respecto a las cuestiones Q1 y Q6, fue la respuesta Q3 (Tabla 1, Figura 3). Reforzar y fomentar la conexión existente entre diferentes disciplinas y la simbiosis entre diferentes asignaturas del master de acuicultura. 
Respecto a la aportación de estas asignaturas en el desarrollo del proyecto, como se puede observar en la Figura 1, los alumnos consideran que se han aplicado los contenidos de otras asignaturas (respuesta Q3), aunque la puntuación ha sido de 3,62 sobre 5, puntuación significativamente menor respecto a las cuestiones Q1 y Q6 (Tabla 1, Figura 3) planteadas a los alumnos. La razón de la menor puntuación se ha obtenido de las respuestas abiertas que han incluido los alumnos. Según indican en las encuestas, hay otras asignaturas que se podrían incluir en el proyecto, como patología, o bien calidad del agua. Sin embargo, al tratarse de un master Interuniversitario y pertenecer estas dos asignaturas a la Universidad de Valencia, parece más complicado la coordinación de éstas dentro del proyecto.

Por otra parte, los alumnos valoran positivamente la metodología aplicada para el aprendizaje y expresan que han cubierto sus expectativas como estudiantes al compararla con otros métodos tradicionales (Figura 3, respuesta Q4). Además, los alumnos expresan una alta valoración cuando se les plantea si la metodología es útil para aplicar los conocimientos vistos en clase para solucionar problemas del proyecto (Figura 3, respuesta Q5).

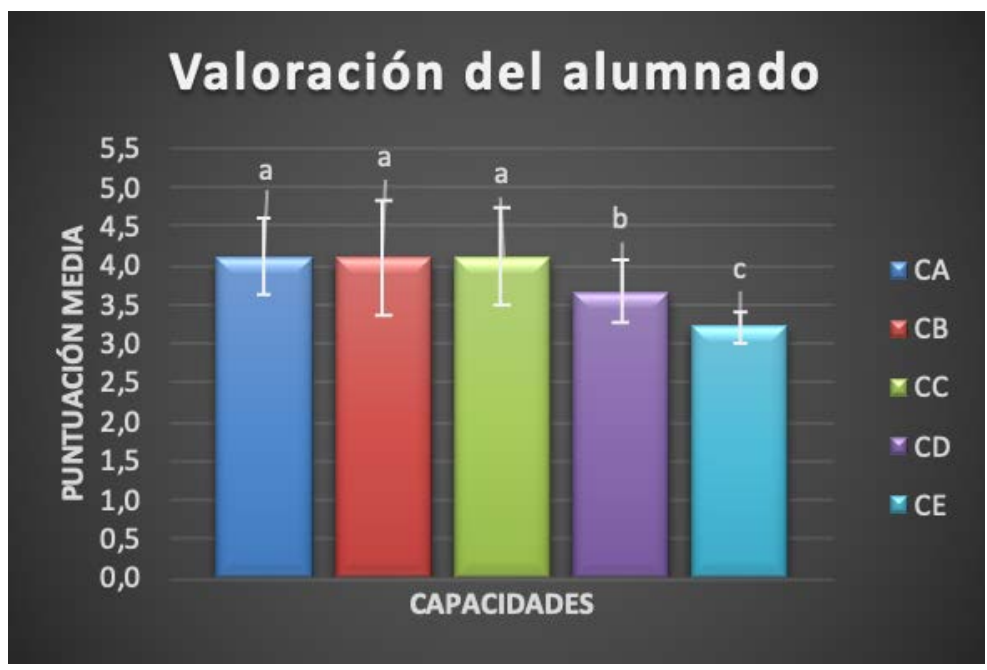

Figura 1. Valoración media de los alumnos, respecto al desarrollo de capacidades a través del ABPr*. Los datos que aparecen en el gráfico son la media \pm desviación estándar $(n=10)$. Letras diferentes en la barras indican diferencias estadísticas significativas con una $P<0,05$.

*Cuestiones planteadas se pueden consultar en la Tabla 1

En relación con el estímulo de los estudiantes en el desarrollo de habilidades para resolver situaciones reales, fue una de las metas más difíciles de alcanzar en el proyecto, principalmente por falta de tiempo necesario para la búsqueda de información. Los alumnos debían de buscar información de una especie en concreto para desarrollar el proyecto. Sin embargo, a pesar de ser un objetivo complicado de alcanzar sí se ha podido valorar por parte de alumnos, que tienen la misma opinión que el profesorado: Falta de tiempo para el desarrollo del proyecto y baja capacidad de planificación, tal y como se puede observar en la Figura 4, respuesta CD.

Durante el desarrollo del proyecto se trabajó en grupo, fomentando las sinergias y complementando las capacidades de las personas que los integraban. Esto se evidencia con la excelente valoración de la capacidad adquirida para el trabajo en grupo y el trabajo autónomo. También es así de valorado por los alumnos (Figura 4 respuestas CA y CB). Sin embargo, los alumnos han visto que el ABPr, no ha contribuido considerablemente a adquirir una mayor capacidad de expresarse adecuadamente, debido a que el tiempo para ello, ha sido reducido cuestión (CD, Figura 4). 
Por lo tanto, los principales aspectos a mejorar en los próximos cursos son:

- Planificación del tiempo para desarrollar el proyecto. Los alumnos y los profesores pensamos que el tiempo para este proyecto es limitado si se quiere desarrollar todo lo planteado.

- Mejorar o fomentar la capacidad de diseño del proyecto por parte de los alumnos. Esto es algo que se puede mejorar a través de la tarea 2 "Braimstorming", junto con una mejor planificación del tiempo. Quizás empezar el proyecto desde las asignaturas "bases".

- Uno de los puntos más difíciles de abordar es la inclusión de otras asignaturas del master que se complementaría muy bien en el proyecto. Este es el caso, por el ejemplo la asignatura patología, o bien calidad del agua, que sería muy útil a la hora de estimar mortalidades y/o tratamientos para una especie piscícola. Sin embargo, esto es muy complicado, pues al tratarse de un master Interuniversitario, complica la coordinación del proyecto con la otra universidad, por lo que hemos decidido, no incluirlo en este primer curso del proyecto.

Como puntos fuertes se deben de subrayar

- Seguimiento personalizado de los alumnos: Uno de los puntos fuertes, a la hora de aplicar el ABPr, que tiene el Master interuniversitario de acuicultura, es que el número de alumnos es menor de 20. Esto supone un control muy alto de los grupos y poder formar grupos de pocas personas (2 alumnos), así como una evaluación muy personalizada de todo el proceso. Sin embargo, el bajo número de alumnos supone una limitación del "output" por parte de estos.

- Elevado enfoque empresarial del proyecto. Debido a la estructura del master de acuicultura, y estando una parte de éste muy enfocado a nivel empresarial, el proyecto planteado se acerca mucho a las situaciones reales. Este hecho incentiva mucho a los alumnos, que ven en el desarrollo de este proyecto una aplicabilidad.

- Capacidad para aplicar la TIC y mejorar la planificación. Debido a que en la UPV disponemos de una plataforma para aplicar las TIC, éstas pueden facilitar una docencia más interactiva, pudiendo fomentar la comunicación e interacción con diversos profesores, y una mejor planificación del tiempo.

- El sector acuícola. El hecho de que este máster sea tan especializado hace que los alumnos matriculados tengan una alta motivación desde el inicio, ya que la mayoría de ellos ingresan en el master siendo éste su primera elección. Esto facilita al profesorado y también motiva la implementación de nuevas mejoras.

- Elevado contacto con el sector acuícola. Durante el desarrollo del proyecto, los alumnos tienen la posibilidad de ponerse en contacto durante el desarrollo de diferentes asignaturas, especialmente en la de producción de especies, con diversos profesionales procedentes de empresas y de centros de investigación de alto prestigio como el CSIC o el IEO, por lo que permite integrar los conocimientos necesarios para la elaboración del proyecto.

Una de las mejoras en la docencia a destacar en el presente ABPr, ha sido una mayor motivación por parte de los alumnos, la cual no solo ha sido medible por las encuestas realizadas, sino también porque se ha registrado la asistencia y durante todo el segundo cuatrimestre solo hubo un día que faltó un alumno, y fue por una causa justificada. También se ha observado una continua participación de los alumnos durante las clases, así como una mejor disposición para realizar las tareas. Otra de las mejoras ha sido el fomentar aprendizaje colaborativo, permitiendo a los estudiantes compartir ideas entre ellos y trabajar activamente con los profesores, expresar sus propias opiniones y negociar soluciones, habilidades todas, necesarias en los futuros puestos de trabajo. Esto también ha promovido que el alumno adquiera responsabilidad en el propio aprendizaje. 


\section{Conclusiones}

La experiencia durante el primer curso de la aplicación del ABPr ha sido muy positiva desde el punto de vista de la motivación y participación de los alumnos, así como en el trabajo colaborativo. No obstante, se deben de mejorar algunos aspectos como es la planificación del tiempo y consolidar relaciones de integración o la interacción entre diferentes disciplinas/asignaturas de manera que tengan una mayor representación en el proyecto, con un enfoque más multidisciplinar.

\section{Referencias}

BERMELL, S. G., MARTON, I., VILLANUEVA, J. F., SÁNCHEZ, A., CARLOS, S. (2019). Aprendizaje basado en proyectos en el Grado en ingeniería de la energía. In IN-RED 2019: V Congreso de Innovación Educativa y Docencia en Red.

ESTRUCH, V., SILVA, J. (2006). Aprendizaje basado en proyectos en la carrera de Ingeniería Informática. Actas de Las XII Jornadas de La Enseñanza Universitaria de La Informática (JENUI, 2006). Deusto, Bilbao, Del, 12, 339-346.

FERNÁNDEZ, F. H., DUARTE, J. E. (2013). El aprendizaje basado en problemas como estrategia para el desarrollo de competencias específicas en estudiantes de ingeniería. Formación Universitaria, 6(5), 29-38.

GAYO, J. E. L., LANVIN, D. F., SALVADOR, J. C., DEL RÍO, A. C. (2006). Una experiencia de aprendizaje basado en proyectos utilizando herramientas colaborativas de desarrollo de software libre. Dpto. de Informática Universidad de Oviedo C/Calvo Sotelo S/N CP, 33007.

JOVER, M., MARTÍNEZ, S., TOMÁS, A., PÉREZ, L. (2016). Propuesta metodológica para el diseño de instalaciones piscícolas. Revista AquaTIC, (19).

LÓPEZ, M. S., VÁZQUEZ, O. L. V. (2015). Aprendizaje Colaborativo basado en proyectos desarrollados en Ingeniería. Revista Iberoamericana Para La Investigación y El Desarrollo Educativo ISSN: 2007-2619, (10).

MERINERO, S., LLORENS, S. M., VIDAL, A. T., CERDÁ, M. J. (2016). Análisis económico de alternativas de producción de Dorada en jaulas marinas en el litoral Mediterráneo español. Revista AquaTIC, (23).

NOGALES MÉRIDA, S., JOVER CERDÁ, M., MARTíNEZ LLORENS, S., TOMÁS VIDAL, A. (2011). A study of partial replacement of fish meal with sunflower meal on growth, amino acid retention, and body composition of sharpsnout seabream, Diplodus puntazzo (Actinopterygii: Perciformes: Sparidae). Acta Ichthyologica et Piscatoria, 41(1). https://doi.org/10.3750/AIP2011.41.1.07

REVERTE BERNABEU, J., GALLEGO SÁNCHEZ, A. J., MOLINA-CARMONA, R., SATORRE CUERDA, R. (2007). El aprendizaje basado en proyectos como modelo docente. Experiencia interdisciplinar y herramientas groupware.

RODRÍGUEZ-SANDOVAL, E., CORTÉS-RODRIGUEZ, M., VARGAS-SOLANO, É. M., LUNA-CORTÉS, J. (2010). Evaluación de la estrategia pedagógica "aprendizaje basado en proyectos": percepción de los estudiantes. Educación y Educadores, 13(1), 13-25. https://doi.org/10.1590/S1414-40772010000100008

SÁNCHEZ, J. (2013). Qué dicen los estudios sobre el Aprendizaje Basado en Proyectos. Actualidad Pedagógica.

VEGA, F., PORTILlO, E., CANO, M., NAVARRETE, B. (2014). Experiencias de aprendizaje en ingeniería química: diseño, montaje y puesta en marcha de una unidad de destilación a escala laboratorio mediante el aprendizaje basado en problemas. Formación Universitaria, 7(1), 13-22. 


\section{Agradecimientos}

Este trabajo se ha desarrollado dentro del marco del proyecto de innovación Educativa: "Aprendizaje basado en proyectos para fomentar la visión profesional en el máster de Acuicultura (B74/18)" "Aprendizaje + Docencia" (A+D), impulsada por el Vicerrectorado de Estudios, Calidad y Acreditación y el Vicerrectorado de Recursos Digitales y Documentación de la Universitat Politècnica de València. 